\title{
Cambios Morfológicos en la Regeneración del Tendón Calcáneo de Rata (Sprague Dawley) por la Aplicación de Ultrasonido Continuo
}

\author{
Morphologic Changes of the Regeneration in the Rat's (Sprague Dawley) \\ Calnaeous Tendon by Continous Ultrasound Applications
}

"Carolina Schencke \& **Mariano del Sol

SCHENCKE, C. \& DEL SOL, M. Cambios morfológicos en la regeneración del tendón calcáneo de rata (Sprague Dawley) por la aplicación de ultrasonido continuo. Int. J. Morphol., 28(2):601-608, 2010.

RESUMEN: Las lesiones de los tendones plantean siempre grandes problemas clínicos y terapéuticos, por su importancia funcional y su peculiar cicatrización. El apoyo fisiátrico, específicamente el tratamiento con ultrasonido, ha demostrado ser eficiente en la regeneración del tendón calcáneo. Este trabajo tuvo como objetivo analizar morfológicamente los efectos del uso de ultrasonido continuo en la regeneración post quirúrgica del tendón calcáneo de rata. Se utilizaron 15 ratas albinas, Sprager Dawle. Luego de 48 horas post tenorrafia se comenzó con una sesión diaria de ultrasonido terapéutico con una intensidad de $1 \mathrm{~W} / \mathrm{cm}^{2}$, frecuencia de $3 \mathrm{MHZ}$, cabezal de $0,5 \mathrm{~cm}$ de ERA, por 30 segundos durante 10 días. Las ratas fueron sacrificadas 48 horas después de terminar las aplicaciones de ultrasonido para la extracción y disección del tendón calcáneo del miembro posterior derecho, mientras que el tendón del miembro posterior izquierdo sirvió de control normal. Para fines de comparación morfológica se trabajó con un grupo de ratas control del tratamiento, en el que se provocó la lesión sin aplicar ultrasonido. Las muestras fueron fijadas en formalina tamponada y teñidas con H.E. Los tendones del grupo experimental se encontraron en un estado de regeneración avanzada, cuyos fibroblastos presentaron características morfológicas propias de una célula activa en la síntesis y secreción de matriz extracelular, con un promedio de fibroblastos similar al obtenido en el tendón normal. A su vez, el tendón del grupo sin tratamiento presentó un estado de regeneración tardío. Las fibras colágenas estaban invadidas por tejido conjuntivo y vasos sanguíneos, con un promedio de fibroblastos que triplica al promedio encontrado en el control normal, lo que determinó que el tendón sin aplicación de ultrasonido se encontrara aún en proceso inflamatorio. Este estudio confirma que el uso de ultrasonido continuo genera una rápida proliferación celular en el tendón lesionado de rata, y en consecuencia, acelera su regeneración.

PALABRAS CLAVE: Tendón calcáneo; Ultrasonido; Morfología; Regeneración tendinosa.

\section{INTRODUCCIÓN}

En el Hombre el tendón calcáneo es el más grueso y resistente del organismo, sin embargo sus lesiones son bastante frecuentes.

Benjamin \& Ralphs, 2000, describen en su estudio sobre bases biológicas del desarrollo de tendones y ligamentos, que las propiedades físicas y de viscoelasticidad de éste dependen de su constitución bioquímica, como es su contenido en agua, cantidad de fibras, presencia de proteoglicanos y colágena, que varía con la edad.

El tendón, en general, es una estructura alargada, de gran resistencia tensil. Se constituye por fibras de colágeno Tipo I, envueltos por un tejido conjuntivo laxo, por donde discurren vasos, linfáticos y fibas nerviosas, denominado endotenon el que se continúa con el peritendon que rodea al tendón externamente. La continuidad del tendón con el músculo se consigue por prolongación del endomisio, perimisio y epimisio con el endotendón y peritendón, y la inserción en el hueso por penetración de las fibras tendinosas hasta conectar con las fibras colágenas óseas. Entre las fibras se dispone un escaso número de células, los tenocitos, que son fibrocitos adaptados a los espacios interfibrilares, con morfología aplanada, alargada o cuadrangular.

\footnotetext{
" Facultad de Ciencias de la Salud, Universidad Autónoma de Chile, Temuco, Chile.

** Facultad de Medicina, Universidad de La Frontera, Temuco, Chile.
} 
Dahlgren, 2007 realizó estudios sobre el daño y reparación de ligamentos y tendones. Este autor describió la morfología del tendón, caracterizados por células fibroblásticas incluidas en un complejo estructural rico en fibras colágenas, organizadas a lo largo de la línea de tensión. Esquema organizacional que imparte las propiedades mecánicas necesarias para su función, estableciendo uniones estables para el sistema músculo esquelético.

Combalía (1994), en su tesis doctoral sobre el estudio estructural y ultraestructural de la rotura del tendón calcáneo, describe al fibroblasto, forma activa del fibrocito, con una ligera basofilia citoplasmática debido al aumento del retículo endoplasmático rugoso (RER), con núlceo ovoide y ligeramente aplanado, cromatina finamente granular y nucléolo pequeño. La síntesis de fibras colágenas sucede dentro y fuera de esta célula. Después de que el procolágeno es secretado hacia el espacio extracelular, el trabajo de ciertas enzimas logra la formación de tropocolágeno, el que se une para formar el colágeno. Los fibroblastos se encuentran anclados a elementos de la matriz extracelular. Estas uniones son importantes para la célula, permitiendo extender prolongaciones citoplasmáticas y moverse.

Sharma \& Maffulli, 2005, señalaron que se distinguen dos tipos de tendones, extrasinoviales e intrasinoviales. Los extrasinoviales son de recorrido rectilíneo y corto, como el tendón calcáneo. Estos presentan un sistema de deslizamiento simple constituido por un tejido conjuntivo laxo que le separa de las estructuras vecinas, denominado paratendón, con suficiente laxitud para permitir el deslizamiento del tendón. A través de esta estructura llega la vascularización al tendón. El tendón tiene vasos sanguíneos y linfáticos íntimamente ligados al trayecto de las arterias. Los nervios se reducen a fibras simpáticas perivasculares y solo la unión musculotendinosa contiene corpúsculos de Golgi y terminaciones neurosensitivas en relación con el control de la tensión.

Las lesiones de los tendones plantean siempre grandes problemas clínicos y terapéuticos, por su gran importancia funcional y su peculiar cicatrización. Según Deydén \& Tejeda (2006), la ruptura de tendón calcáneo ocupa el tercer lugar de las lesiones mayores de tendones en humanos, después del manguito rotador y del mecanismo extensor. La edad de mayor incidencia de este tipo de lesiones encuentra entre los 30 y 50 años de edad, se estima que la lesión puede no ser diagnosticada hasta en un 25\% de los casos, como describe Enriquez et al. (2002). En la mayoría de los casos el tendón afectado es difícil de reparar, así como el manejo de las lesiones crónicas. El apoyo fisiátrico ha demostrado ser eficiente como tratamiento alternativo. El ultrasonido continuo ha sido usado como tratamiento com- plementario en daños a nivel de ligamentos, músculo y tendón, (Kitchen \& Patridge, 1990).

En los últimos años se ha observado que mediante estímulos físicos no invasivos como el ultrasonido se puede acelerar el proceso de reparación de las lesiones de tendón calcáneo. La energía acústica generada por el ultrasonido es de baja frecuencia, se transmite por los tejidos a través de vibración molecular. Según Dalecki (2004), el ultrasonido de baja intensidad, 0.5 -3000, es utilizado en terapia física, mientras que el de alta intensidad es utilizado en tratamiento de tumores generando necrosis. La absorción del ultrasonido resulta en conversión de energía al calor. El efecto es de aproximadamente $1^{\circ} \mathrm{C}$, al cual enzimas como metaloproteinazas o colagenasa son muy sensitivas a pequeñas variaciones de temperatura, restableciendo el normal efecto metabólico (Kamakura, 1995). La exposición al ultrasonido estaría promoviendo el incremento de señales extracelulares, kinasas, activación de síntesis de DNA, y como consecuencia, proliferación celular.

Algunos estudios comunican daño y alteraciones a nivel cromosómico cuando este tratamiento se ocupa en tejidos blandos sin alteración o que no hayan sido lesionados, (Deyne \& Kirsch, 1995). Grass et al., 1984; Roebroeck, et al., 1998, observaron que el uso de este tratamiento si es efectivo en la regeneración de tejidos, mostrando un efecto de aceleración en el índice mitótico, recomendando especial cuidado en la elección del sitio de aplicación tanto como en la duración. Bassoli, 2001, en su estudio sobre los efectos del ultrasonido continuo en la regeneración del músculo esquelético demostró que el área estimulada era capaz de inducir mitosis en células satélites. Observaron aceleración en la regeneración de fibras musculares, mioblastos y agregados de miotubos.

En análisis inmunocitoquímicos con PCNA (Tsai et al., 2005), confirmaron que el ultrasonido pulsátil estimula la proliferación celular en el proceso de regeneración de tendón calcáneo. Papatheodorou et al., 2009, observaron una aceleración en la generación de fibras colágenas con ultrasonido pulsátil cuando este es aplicado tempranamente en el proceso de cicatrización del tendón.

La escasa información en relación a los cambios morfológicos que conlleva la rapidez en la regeneración del tendón luego de la aplicación de ultrasonido continuo fue un factor que llevó a emprender el siguiente estudio.

Esta investigación tiene como finalidad demostrar experimentalmente los efectos del ultrasonido continuo en la morfología del tendón calcáneo de rata, pudiendo ser aplicable en la clínica fisioterapéutica. 


\section{MATERIAL Y MÉTODO}

Con el objetivo de analizar morfológicamente el efecto de la aplicación de ultrasonido continuo en una lesión de tendón calcáneo se utilizaron 15 ratas albinas, Sprager Dawley. Para fines de comparación morfológica se trabajó con tres grupos de animales, grupo experimental, control y sin tratamiento. En el grupo experimental se utilizó el tendón del miembro posterior derecho, mientras que el tendón del miembro posterior izquierdo sirvió de control. En el grupo sin tratamiento se provocó la lesión sin aplicar ultrasonido. Se depiló el miembro posterior derecho con crema depilatoria Veet. Para efectos de anestesia local se realizó una punción en la zona del tendón calcáneo y nervio isquiático con lidocaina al $2 \%$ más epinefrina. Luego de un minuto se realizó una incisión paralela al tendón. Se disecó la zona para exponer el tendón. El tendón se levantó con una pinza anatómica y se pasó un punto para evitar la retracción del tendón luego de su sección. Se realizó el corte del tendón calcáneo a 5 milímetros proximal a su inserción en el hueso y se suturó con vicril. Para la sutura de la piel se utilizó seda negra trenzada. Se aplicaron de 2 a 3 puntos de sutura de acuerdo al tamaño de la lesión quirúrgica.

Luego de 48 horas post tenorrafia se comenzó con una sesión diaria de ultrasonido terapéutico continuo, marca enraf-nonius modelo sonopuls 590 con una intensidad de $1 \mathrm{~W} / \mathrm{cm}^{2}$, frecuencia de $3 \mathrm{MHZ}$, cabezal de $0,5 \mathrm{~cm}^{2}$ de ERA, por 30 segundos durante 10 días. Las ratas fueron sacrificadas 48 horas después de terminar el tratamiento para la extracción y disección del tendón calcáneo, el cual fue colocado en formalina tamponada al $10 \%$. Las muestras fueron procesadas, deshidratadas en batería de alcoholes y procesadas para su inclusión en paraplast.

Posteriormente, se realizaron cortes transversales semiseriados de $7 \mu \mathrm{m}$ de espesor, utilizándose micrótomo rotatorio marca Microm, modelo HM 325. Al llegar al tendón, se realizaron cortes seriados de $5 \mu \mathrm{m}$ de espesor. Los cortes histológicos se montaron en portaobjeto con la ayuda de un baño de flotación marca Lab-line, modelo 25104. Para el estudio morfológico y para las fotografías se utilizó un microscopio Carl Zeiss, Axiolab, con cámara Mc80 DX. Para el análisis morfológico, los cortes fueron teñidos con Hematoxilina-Eosina.

Para determinar el número de núcleos de fibroblastos por $\mathrm{mm}^{2}$ se utilizó el método planimétrico. Para ello, se observaron cinco láminas por cada individuo de cada grupo, analizando 5 campos por lámina, observando un total de 175 campos, considerando los tres grupos.

\section{RESULTADOS}

Los tendones calcáneos del grupo de ratas sin tratamiento y aquellos tratadas con ultrasonido continuo, presentaron un grosor promedio entre 4,5 y $5 \mathrm{~mm}$, respectivamente (Figs. 1A y B).

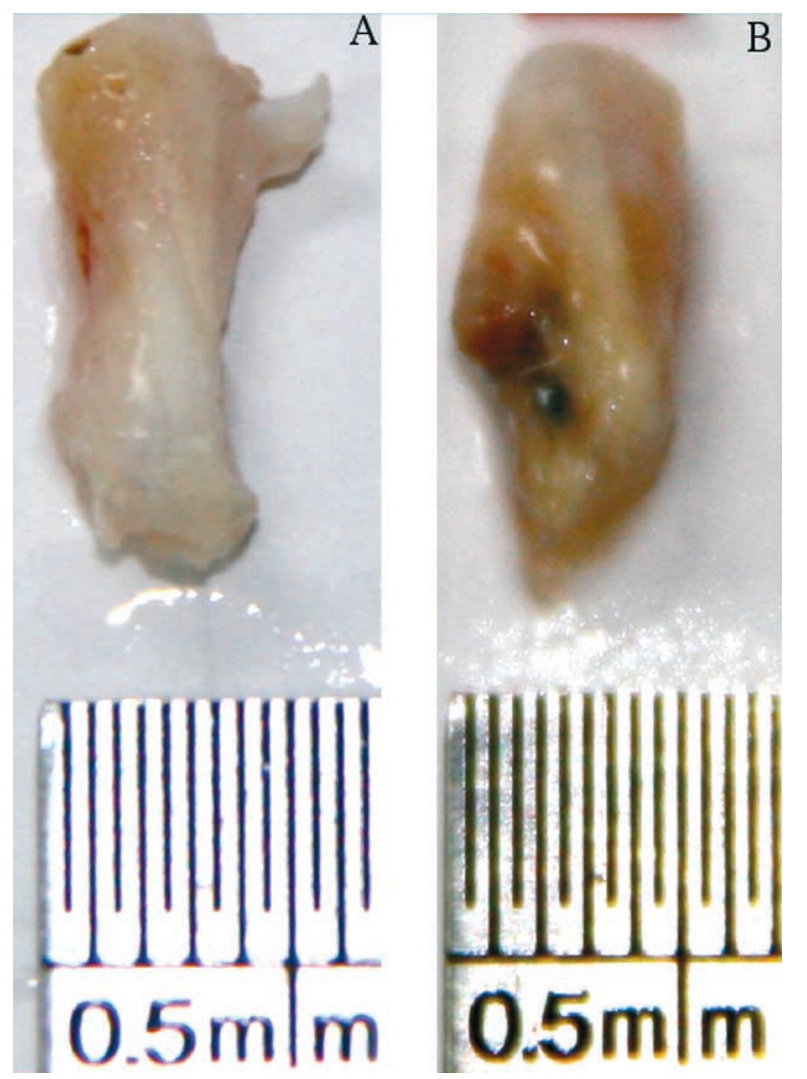

Fig. 1. Tendones calcáneos de rata disecados. A. Tendón sin tratamiento (con lesión y sin aplicación de US). B. Tendón con 10 sesiones de ultrasonido continuo.

Los resultados del análisis histológico de tendones normales de rata presentan fibroblastos embebidos en una matriz extracelular rica en fibras colágenas organizadas a lo largo de la línea de tensión. Los fibroblastos presentan núcleos aplanados, alargados y escasamente teñidos. Estas células se encuentran rodeadas de fibras colágenas alineadas en forma paralela (Fig. 2).

En el grupo de ratas cuyo tendón fue lesionado sin aplicación de ultrasonido continuo, usado como control del tratamiento, presentaron un grosor promedio de 4 a 4,5 $\mathrm{mm}$. En estos tendones se observa una evidente reacción inflamatoria, con tejido conjuntivo y vasos sanguíneos irrumpiendo la región tendinosa (Fig. 3). 


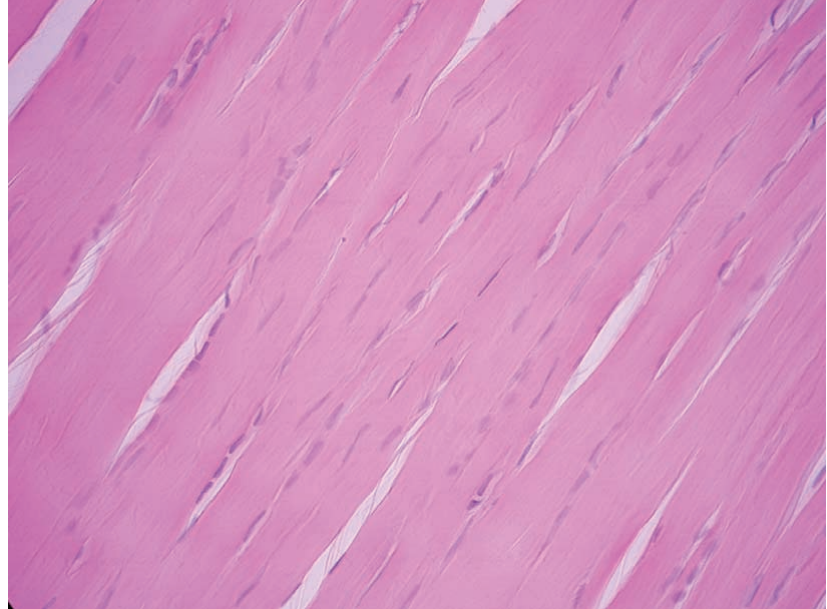

Fig. 2. Tendón control normal de rata. Se observan fibroblastos con núcleo aplanado y alargado (flecha). Rodeando los fibroblastos se encuentran las fibras colágenas dispuestas en forma paralela. HE, 400X.

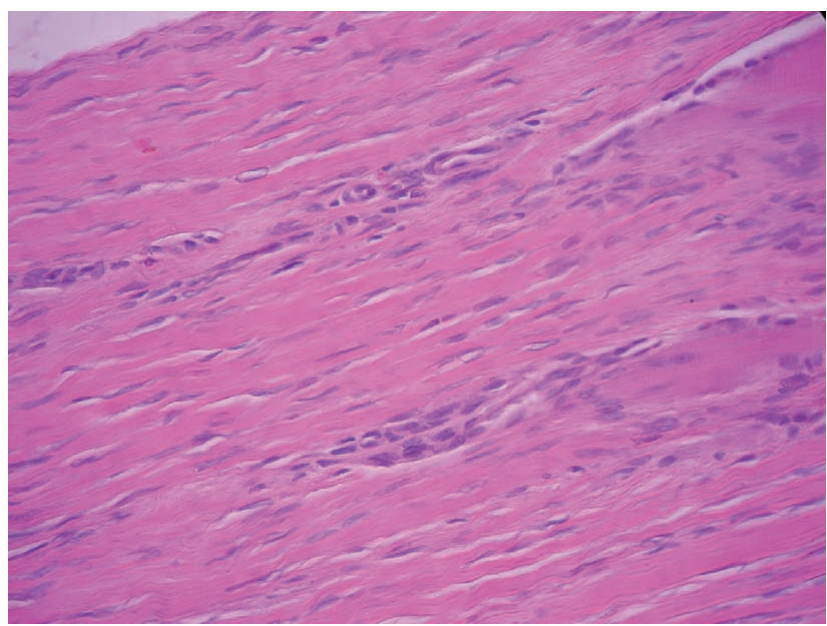

Fig. 3. Tendón calcáneo de rata control del tratamiento (no estimulado con U.S continuo). Se observa una evidente reacción inflamatoria, con tejido conectivo y vasos sanguíneos irrumpiendo la región tendinosa (flechas). HE. 400X.

En el grupo experimental de ratas, cuyo tendón fue estimulado con ultrasonido continuo, se observó un proceso de regeneración avanzado, con características morfológicas similares al tendón normal. Los fibroblastos presentaron núcleo ovoide, ligeramente aplanado y levemente hipertróficos (Figs. 4a,b). En estos tendones se observó la presencia de tejido conjuntivo denso irregular rodeando externamente la zona tendinosa, la que presentó neoformación vascular. El tejido conjuntivo no invadió el tendón calcáneo, como se observó en el grupo sin tratamiento (Figs. 5a,b).
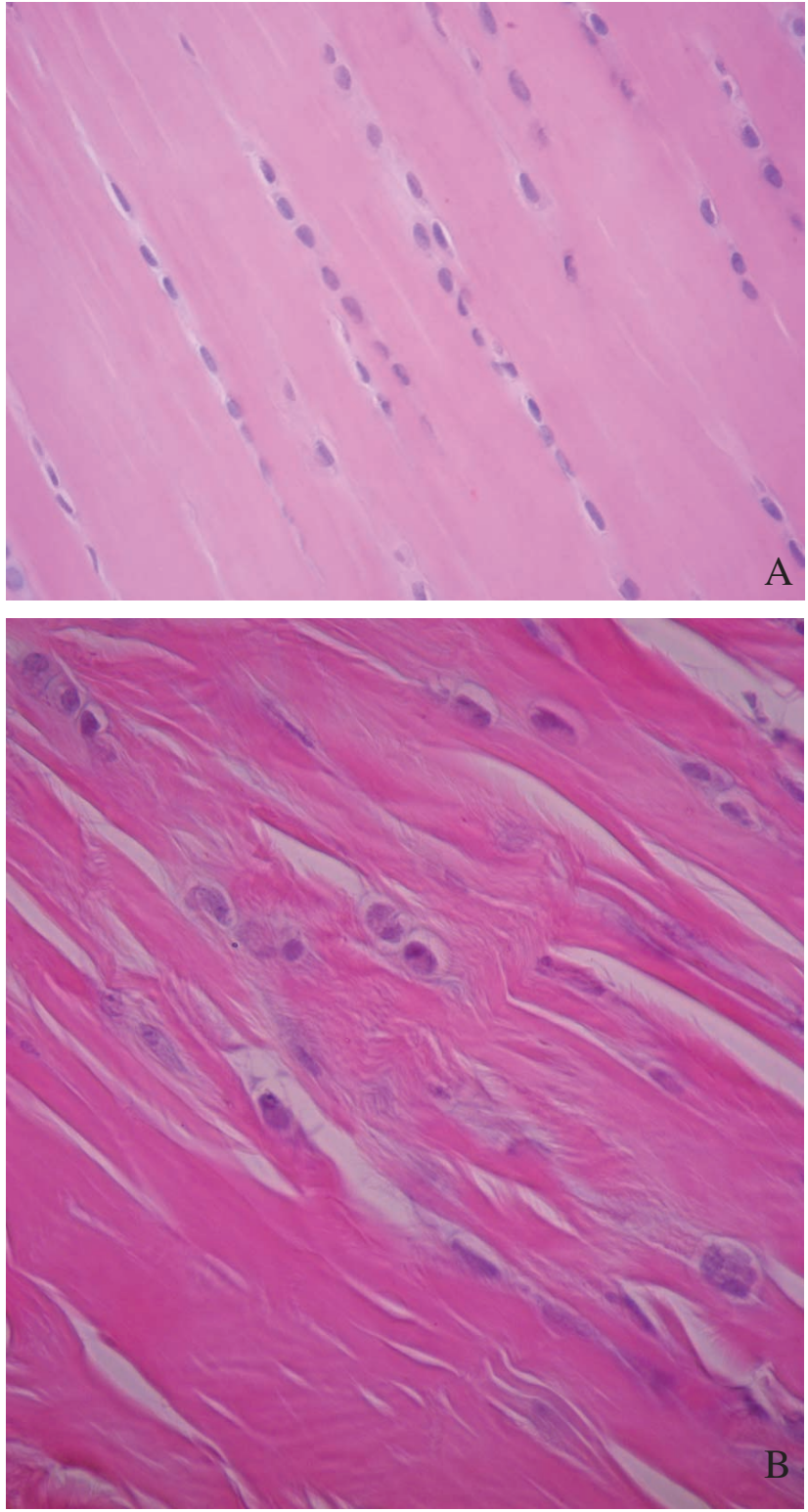

Fig. 4. Tendón calcáneo de rata lesionado, estimulado con U.S. continuo y disecado a los 16 días post tenorrafia. A. El tendón presenta un proceso de cicatrización avanzada, con características morfológicas similares al tendón normal. H.E. 400X. B. Los fibroblastos presentan núcleo ovoide (flecha), 600X. H-E.

En el grupo de ratas experimentales, el promedio de fibroblastos de tendones tratados con ultrasonido continuo fue de 2.440 células por $\mathrm{mm}^{2}$, número que se aproxima al promedio obtenido en el control normal de 2070 células/ $\mathrm{mm}^{2}$. El promedio de fibroblastos obtenido del tendón sin tratamiento con US continuo presentó hipercelularidad, obteniéndose un promedio de 5030 células por $\mathrm{mm}^{2}$, lo que se observa en el Fig. 6. 


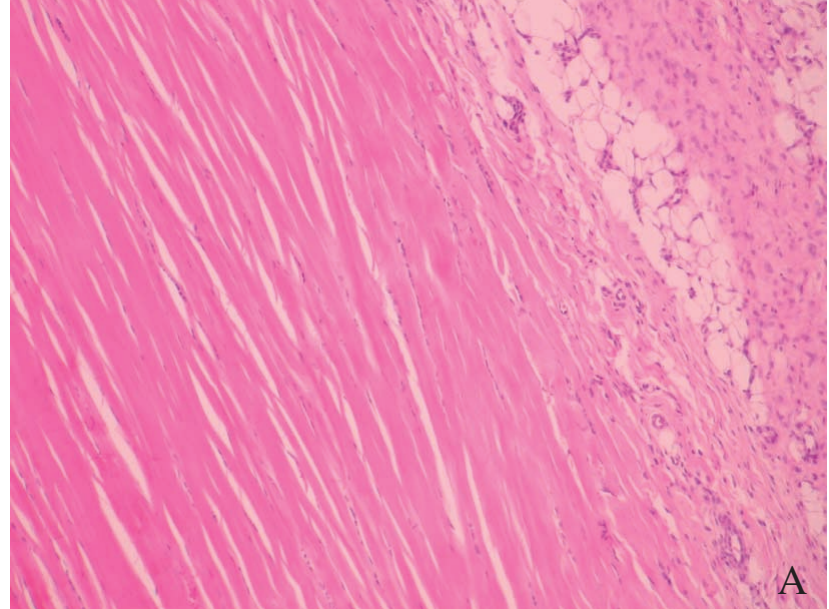

Fig. 5. Tendón calcáneo de rata lesionado, estimulado con U.S. continúo y disecado a los 16 días post tenorrafia. A. El tendón se rodea externamente de tejido conjuntivo denso irregular (flecha). HE. 400X. B. En el tejido conjuntivo que rodea al tendón se observan vasos sanguíneos.

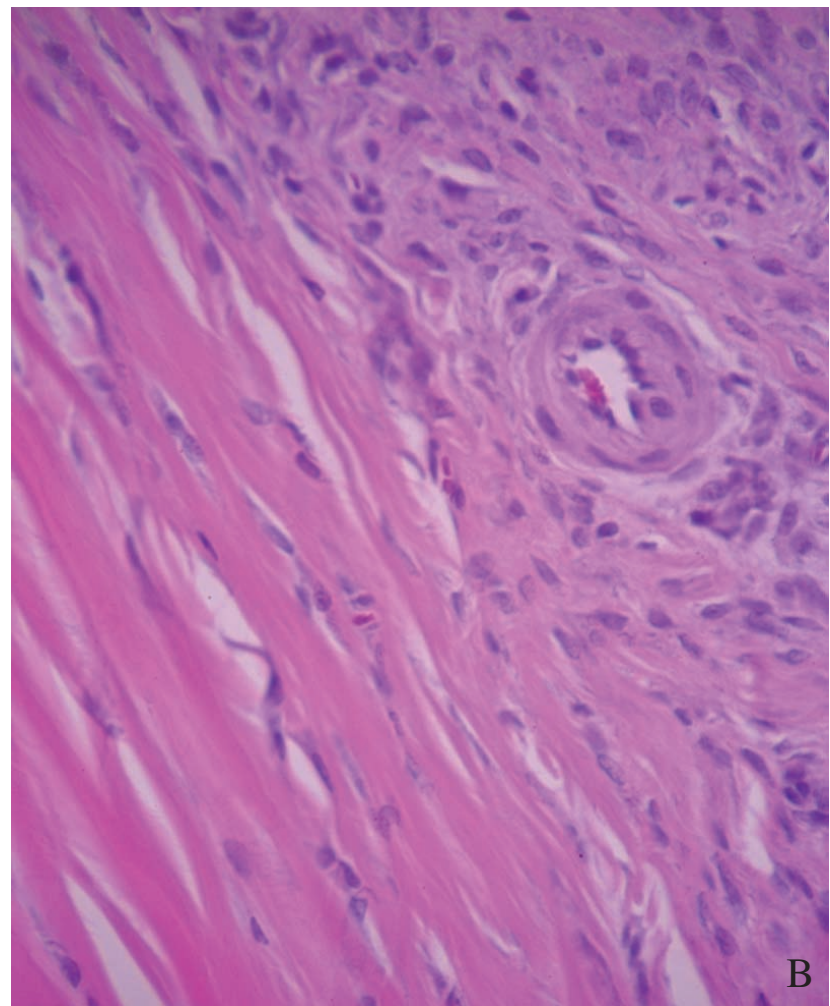

\section{Células $/ \mathrm{mm}^{2}$}

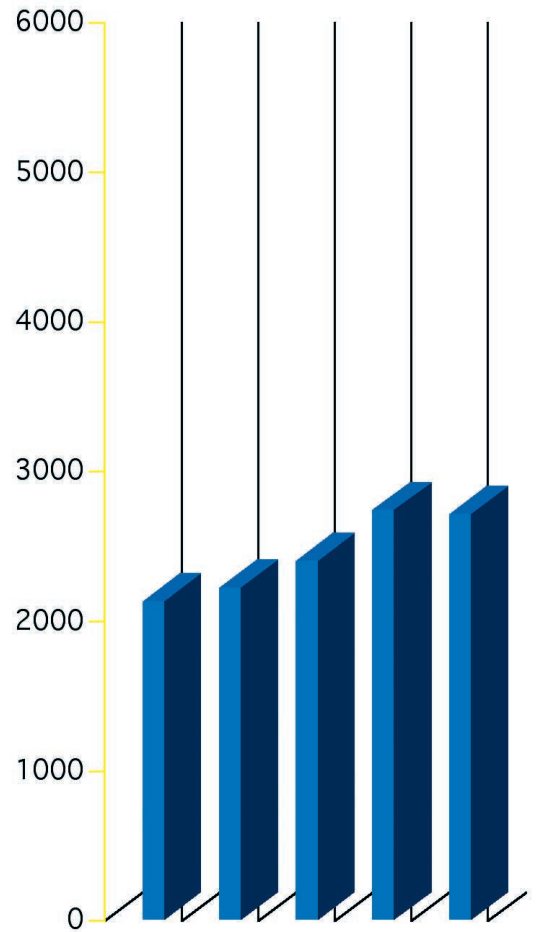

Grupo experimental

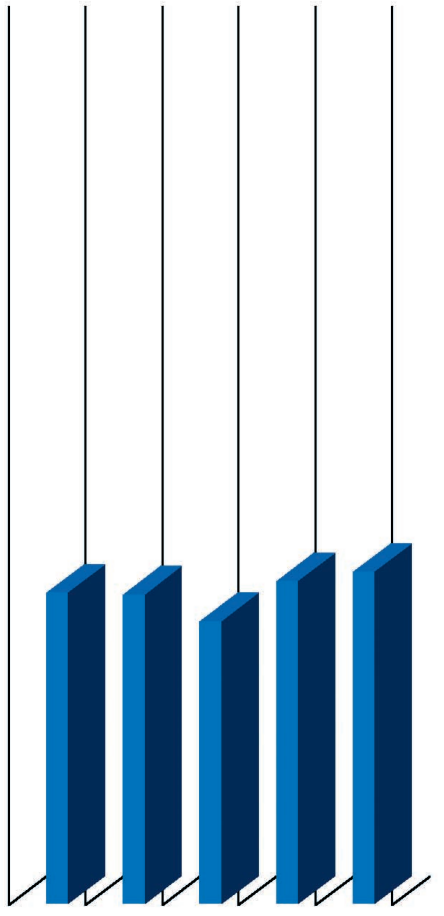

Grupo control

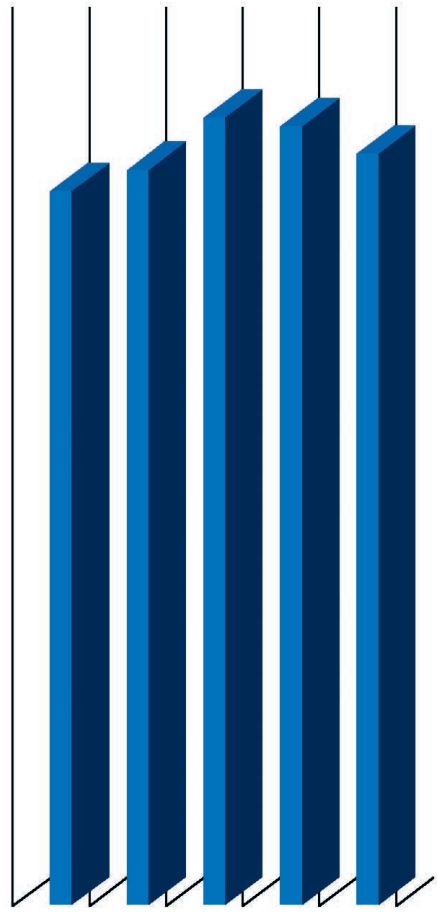

Grupo sin tratamiento

Fig. 6. Promedio de fibroblastos en tendones calcáneos de 15 ratas tratadas con ultrasonido continuo en grupos experimental, control y sin tratamiento. 


\section{DISCUSIÓN}

En el estudio se puede observar que, en el día 16 post tenorrafia, el tendón calcáneo lesionado y tratado con ultrasonido continuo se encuentran en el término del proceso de regeneración tendinosa. Las fibras colágenas se observaron reordenadas, ubicándose en forma paralela, con un promedio de fibroblastos que se aproxima al encontrado en el tendón normal. Sin embargo, los tendones controles del tratamiento (no tratados con ultrasonido continuo), se encuentran en un inminente estado inflamatorio.

Este estudio confirma trabajos previos que describen una aceleración de la regeneración tendinosa gracias al uso de ultrasonido (Grass et al.; Bassoli; da Cunha et al., 2001; Berná-Serna et al., 2005; Yeung et al., 2006). Por otro lado, se demuestra la presencia de fibroblastos activos en la secreción de matriz extracelular con una rápida proliferación celular, lo que lleva, finalmente, a una aceleración de la regeneración tendinosa, posterior a una lesión.

En los tendones calcáneos de rata tratados con ultrasonido continuo se observó una neoformacion de vasos sanguíneos en el tejido conjuntivo que rodea al tendón, o peritendon. Este tejido no irrumpe la región tendinosa, como se observó en el tendón control del tratamiento. De esta forma, concordamos con los resultados obtenidos por Nghiem Doan et al., 1999; Mizrahi et al., 2007, quienes describen in vitro resultados controlados y no invasivos de regeneración, proliferación celular y angiogenesis luego de la aplicación de ultrasonido continuo.

Khanna et al., 2009, en su estudio sobre el efecto del ultrasonido en tejidos blandos, indica que falta estan- darizar las dosis del tratamiento según necesidades de cada tejido. La frecuencia de ultrasonido elegida para aplicar al tendón calcáneo de rata en estudio se valida con investigaciones previas que determinan una intensidad ideal en el rango de 1.0 a $2.0 \mathrm{~W} / \mathrm{cm}^{2}$. De Oliveira et al., 2008, demuestran que las propiedades biofísicas del ultrasonido pulsatil aceleran el proceso de proliferacion en tejidos biológicos al irradiar en este rango. Así lo demuestra Hsu \& Huang, 2004, quienes trabajan con diversas intensidades de ultrasonido continuo. Al aplicar el rango de 1.6-2.0 W/ $\mathrm{cm}^{2}$ observan cambios en la orientación morfológica de células endoteliales, e incremento de la secreción de matriz extracelular. Las ratas utilizadas en este trabajo no fueron inmovilizadas luego de la tenorrafia, coincidiendo con $\mathrm{Ng}$ et al., 2004, quienes sugieren el uso de ultrasonido terapéutico de $2 \mathrm{~W} / \mathrm{cm}^{2}$ con posterior terapia de ejercicio, logrando así, aumentar la fuerza tensil en la reparación del tendón calcáneo de rata. Estudios realizados por $\mathrm{Ng} \&$ Fung, 2007; Ng et al., 2003, indican que los resultados de reparación del tendón calcáneo con ultrasonido pulsátil no varían de forma importante en el rango de 1.0 a $2.0 \mathrm{~W} /$ $\mathrm{cm}^{2}$. Sugieren que tanto intensidades altas como bajas de aplicación de ultrasonido pulsátil aceleran el proceso de reparación en tendón calcáneo.

Desde el punto de vista funcional, la reparación tendinosa se manifiesta por su fuerza, elasticidad, y capacidad de energía de absorción, parámetros que deberán ser examinados y comparados con los resultados morfológicos en estudios posteriores.

En conclusión, el presente estudio confirma que el uso terapéutico del ultrasonido continuo en tendón calcáneo de rata genera proliferación de fibroblastos y su activación en la secreción de MEC, en consecuencia, acelera la regeneración tendinosa, posterior a una lesión.

SCHENCKE, C. \& DEL SOL, M. Morphologic changes of the regeneration in the rat's (Sprague Dawley) calnaeous tendon by continous ultrasound applications. Int. J. Morphol., 28(2):601-608, 2010.

SUMMARY: Tendon injuries always account for major clinical and therapeutic problems, due to their functional significance and unique healing. Physiatrics support, specifically ultrasound treatment, has been shown to be effective in the regeneration of the calcaneal tendon. The objective of this study was to morphologically analyze the effect of the use of continuous ultrasound in the post surgery regeneration of the calcaneal tendon of the rat. Ten albino, Sprager Dawley rats were used. Following 48 hours of post tenorrhaphy, a daily session of therapeutic ultrasound was initiated, with an intensity of $1 \mathrm{~W} / \mathrm{cm}^{2}$, frequency of $3 \mathrm{MHZ}$, header at $0.5 \mathrm{~cm}$ ERA, per 30 seconds for 10 days. The rats were sacrificed 48 hours after completion of the ultrasound treatments, for the extraction and dissection of the calcaneal tendon of the right posterior member, while the left posterior member was used for normal control. For purposes of morphological comparison a group of control rats for the treatment was used, in which the injury was caused without applying the ultrasound. Samples were fixed in buffered formalin and stained with H.E. The tendons of the experimental group were found to be in an advanced stage of regeneration which fibroblasts presented morphological characteristics of an active cell in the synthesis and secretion of the extracellular matrix, with an average of fibroblasts similar to that obtained in the normal tendon. At the same time, the tendon of the control group in the treatment, presented a later regeneration stage. The collagen fibers were infiltrated by conjunctive tissue and blood vessels, with an average of fibroblasts that tripled the average found in the normal control, which determined that the tendon without the ultrasound treatment was still in an inflammatory process. This study confirms that the use of continuous ultrasound generated a rapid cellular proliferation in the injured tendon of the rat, and consequently accelerates its regeneration.

KEY WORDS: Calcaneous tendon; Ultrasound; Morphology; Tendon regeneration. 


\section{REFERENCIAS BIBLIOGRÁFICAS}

Bassoli, D. Avaliacão dos efeitos do ultra-som pulsado de baixa intensidade na regeneracão de músculos esqueléticos com vistas a aplicabilidade em clínica fisioterapéutica. Tese spresentada para a obtenção do grado de Mestre. Universidade de São Pablo, 2001.

Benjamin, M. \& Ralphs, J. R. The cell and developmental biology of tendons and ligaments. Int. Review of Cytology, 196: 85-130, 2000.

Berná-Serna, J.; Sanchez-Garre, J.; Madrigal, M.; Zuazu, I. \& Berná-Mestre, J. Ultrasound therapy in rectus sheat hematoma. Phys. Ther., 85(4):352-7, 2005.

Combalía Aleu, A. Estudio estructural, ultraestructural y clínico de la rotura espontánea del tendón de Aquiles en el deportista Ciències Morfològiques $i$ Odontoestomatologia Ciències de la Salut. Tesis Doctoral. Universidad de Barcelona, 1994.

Da Cunha, Alessandra; Parizotto, N. \& de Campos Vidal, B. The effect of therapeutic ultrasound on repair of the achilles tendon (tendo calcaneus) of the rat. Ultrasound in Medicine \& Biology. 27(12):1691-6, 2001.

Dalecki, D. Mechanical bioeffects of ultrasound. Annu. Rev. Biomed. Eng., 6:229-48, 2004.

Dahlgren, L. Pathobiology of Tendon and Ligament Injuries. Clinical Techniques in Equine Practice, 6(3):16817, 2007.

Deydén, S. \& Tejeda, M. Lesión aguda del tendón de aquiles. Ortho-Tips., 2(4):270-6, 2006.

Deyne, P. \& Kirsch, M. In vitro effects of therapeutic ultrasound on the nucleus of human fibroblasts. Physiotherapy, 75(7):629-53, 1995.

De Oliveira, R.; Pires Oliveira, D.; Machado, A.; Soares da Silva, N.; Magini, M \& Pacheco-Soares, C. Assessment of fibroblast cells submitted to ultrasonic irradiation. Cell Biology Int., 32(10):1329-5, 2008.

Enríquez, J.; García, A.; Pastrana, F.; López, A.; González, A. \& Romo, F. Tratamiento de las rupturas crónicas del tendón de Aquiles con plastia de ChristensenSilverskjiold utilizando el tejido fibroso como puente. Rev. Mex. Ortop., 16(3):119-23, 2002.
Georgios K. Koukoulis, Vasilios C. Protopappas, Dimitrios I. Fotiadis \& Constantinos Stathopoulos. Effect of Transosseous Application of Low-Intensity Ultrasound at the Tendon Graft-Bone Interface Healing: Gene Expression and Histological Analysis in Rabbits. Ultrasound in Medicine \& Biology. 35(4):576-84, 2009.

Grass, J.; Gaete, I.; Hernández, I.; Fuenzalida, M.; Illanes, J. \& Lemus, D. Estudio de los efectos del ultrasonido en el tejido embrionario in vitro. Rev. Chil. Ped., 54(3):1538, 1984 .

Hsu, S. H. \& Huang, T. B. Bioeffect of ultrasound on endothelial cells in vitro. Biomolecular Engineering, 21(3-5):99-104, 2004.

Kitchen, S. \& Patridge, C. A review of therapeutic ultrasuond: part 1. Backround and physiological effetcs. Physiotherapy, 76:593-9, 1990.

Kamakura, T.; Matsuda, K. \& Kumamoto, Y. Acoustic streeming induced in focused gaussian beams. J. Acoust. Soc. Am., 97:12740-6, 1995.

Khanna, A.; Nelmes, R.; Gougoulias, N.; Maffulli, N. \& Gray, J. The effects of lipus on soft-tissue healing: a review of literature. British med. Bull., 89:169-82, 2009.

Mizrahi, N.; Seliktar, D. \& Kimmel, E. Ultrasound-Induced Angiogenic Response in Endothelial Cells. Ultrasound in Medicine \& Biology, 33(11): 1818-182, 2007.

Nghiem Doan; Reher, P. \& Sajeda. In vitro effects of therapeutic ultrasound on cell proliferation, protein synthesis, and cytokine production by human fibroblasts, osteoblasts, and monocytes. Journal of Oral and Maxillofacial Surgery. 57(4): 409-419, 1999.

Ng, G. Y. F. \& Fung, T. C. The Effect of Therapeutic Ultrasound Intensity on the Ultrastructural Morphology of Tendon Repair. Ultrasound in Medicine \& Biology. 33(11):1750-4, 2007.

Ng, G. Y. F. ; Ng O. Y. C. \& See K. N. E.Comparison of therapeutic ultrasound and exercises for augmenting tendon healing in rats. Ultrasound in Medicine \& Biology, 30(11):1539-43, 2004.

Ng, O. Y. C.; Ng, Y. F. G.; See, K. N. E. \& Leung, C. P. M. Therapeutic ultrasound improves strength of achilles tendon repair in rats. Ultrasound in Medicine \& Biology. 29(10):1501-6, 2003. 
Papatheodorou, L. K.; Malizos, K. M.; Poultsides, L. A.; Hantes, M. E.; Grafanaki, K.; Giannouli, S.; Ioannou, M. G.; Koukoulis, G. K.; Protopappas, V. C.; Fotiadis, D. I. \& Stathopoulos, C. Effect of Transosseous Application of Low-Intensity Ultrasound at the Tendon Graft-Bone Interface Healing: Gene Expression and Histological Analysis in Rabbits. Ultrasound in Medicine \& Biology. 35(4):576-84, 2009.

Roebroeck, M.; Dekker, J. \& Oostendrp, R. The use of therapeutic ultrasound by physical therapists in dutch primary health care. Phys. Ther., 78(5):470-8, 1998.

Sharma, P. \& Maffulli, N. Basic biology of tendon injury and healing. The Surgeon, 3(5): 309-16, 2005.

Tsai, W. C.; Hsu, C. C.; Tang, F. T.; Chou, S. W.; Chen, Y. J. \& Pang, J. H. S. Ultrasound stimulation of tendon cell proliferation and upregulation of proliferating cell nuclear antigen. J. of Orthopaedic Res., 23(4):970-976, 2005.

Yeung, Ch.; Guo, X.; Fat, J. \& Ng, Y. Pulsed ultrasound treatment accelerates the reapair of achilles tendon rupture in rats. J. of Orthopaedic Res., 24(2):193-201, 2006.
Dirección para correspondencia:

Prof. MSc. Carolina Schencke Figueroa

Universidad Autónoma de Chile.

Avda. Alemania 01090

Temuco

CHILE

Email: carolina.schencke@uautonoma.cl

Recibido : 19-11-2009

Aceptado: 22-03-2010 